CANCER

\section{Antitumour metabolism}

Changing the way that tumours metabolize sugar can stall their growth.

When cells convert glucose into energy and molecular building blocks, the last step in the metabolic pathway is catalysed by an enzyme called pyruvate kinase. Dividing cells, such as cancer cells, contain a form of this enzyme called PKM2. Matthew Vander Heiden at the Massachusetts Institute of Technology in Cambridge and his colleagues found that small-molecule activators of PKM2 impaired tumour formation in mice bearing human cancer cells. Tumours developed later and remained smaller in treated mice than in untreated controls.

The activators bind to PKM2 at a previously unknown site. The authors suggest that, in so doing, the activators alter tumour metabolism in such a way as to deplete growth-promoting compounds produced earlier in the pathway.

Nature Chem. Biol. http://dx.doi. org/10.1038/nchembio. 1060 (2012)

NANOTECHNOLOGY

\section{Strong and stable nanocrystals}

A theoretical method could aid in the design of nanocrystalline metal alloys that retain their unusual properties, such as remarkable strength, at high temperatures.

Nanostructured metals, which are made up of individual metal grains less than 100 nanometres in size, are often unstable at high temperatures owing to instabilities arising from

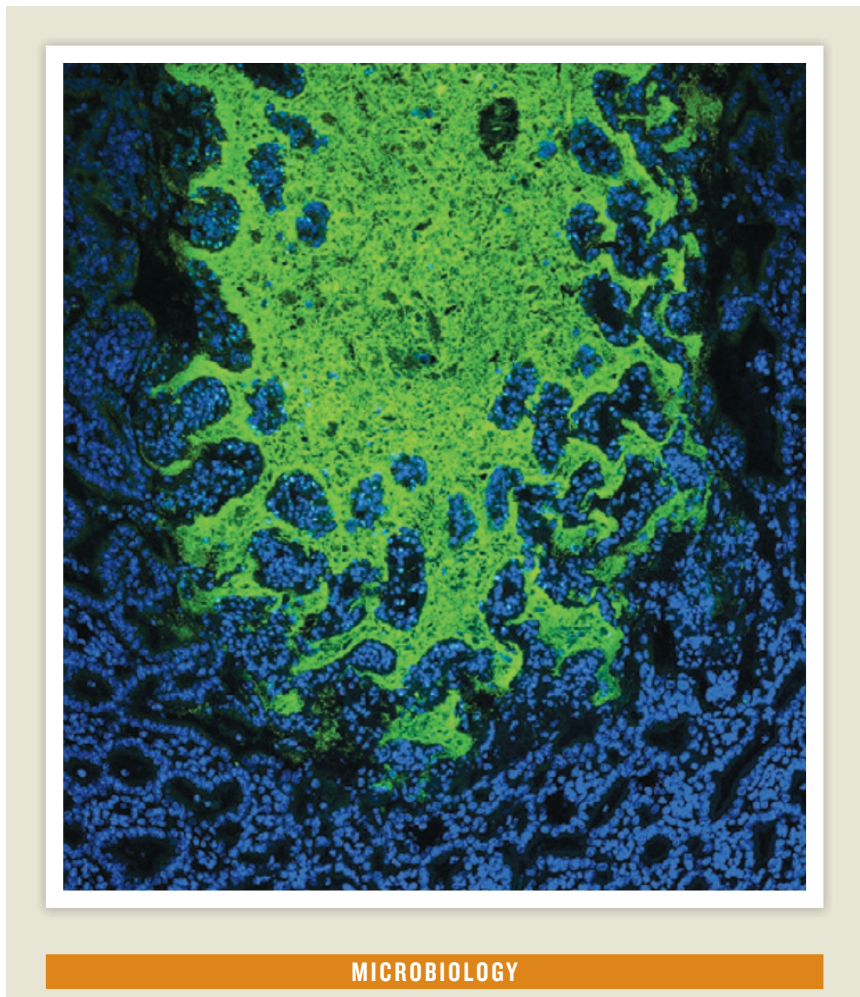

\section{Infection breaks truce}

Gastrointestinal infections can cause the immune system to attack some of the billions of microbes that are normally tolerated in the mammalian gut.

Yasmine Belkaid at the National Institute of Allergy and Infectious Diseases in Bethesda, Maryland, and her team demonstrated this immune response when they infected mice with the parasite Toxoplasma gondii. The infection caused the bacteria normally resident in the gut to breach the gut wall (pictured), activating the animals' immune systems. This triggered immune cells called $\mathrm{T}$ cells to target the gut bacteria. The $\mathrm{T}$ cells then developed into long-lasting memory $\mathrm{T}$ cells - providing an immunological record of the response.

As a result, a bout of food poisoning could have enduring consequences for the immune system, and could even contribute to the development of inflammatory bowel disease, the researchers suggest.

Science http://dx.doi.org/10.1126/science.1220961 (2012) the boundaries between grains. Christopher Schuh and his colleagues at the Massachusetts Institute of Technology in Cambridge developed a theoretical framework for predicting the energy associated with the grain boundaries. The researchers designed and produced an alloy of titanium and tungsten that turned out to be more stable at high temperatures than pure nanocrystalline tungsten, and that could be processed in bulk.

Science 337, 951-954 (2012)

\section{NEURODEVELOPMENT}

\section{Low-flow blood- vessel pruning}

The zebrafish brain's complex network of blood vessels develops not only through vessel growth, but also as a result of pruning, which is driven by changes in blood flow.

Jiu-lin Du at the Chinese Academy of Sciences in Shanghai and his team followed the development of transgenic zebrafish larvae in which cells of the blood vessels, blood and brain carry fluorescent tags. Over the course of six days after fertilization, the researchers imaged the creatures' developing midbrain. They found that, as the network of blood vessels expands, pruning occurs - mainly at loop-shaped segments when cells lining the vessels migrate towards adjacent unpruned segments, resulting in decreased vessel-network complexity. Pruned segments have a lower and more variable blood flow than unpruned ones, and experimental blockade of blood flow triggers the pruning process.

PLoS Biol. 10, e1001374 (2012)

$$
\text { EPIGENETICS }
$$

\section{Clues from baby blood spots}

Blood from the pricked heels of newborn babies can provide insight into the epigenome chemical changes to DNA that influence gene expression before diseases set in.

Most babies born in developed countries have drops of their blood blotted onto a slip of paper called a Guthrie card (pictured), which is used to screen for certain diseases. Vardhman Rakyan and David Leslie at 